\title{
Three-mode optoacoustic parametric interactions with a coupled cavity
}

\author{
H. Miao, C. Zhao, L. Ju, S. Gras, P. Barriga, Z. Zhang, and D. G. Blair \\ School of Physics, University of Western Australia, Western Australia 6009, Australia \\ (Received 2 March 2008; revised manuscript received 6 July 2008; published 4 December 2008)
}

\begin{abstract}
We theoretically analyze three-mode optoacoustic parametric interactions in a coupled Fabry-Perot cavity, where one acoustic mode interacts with two optical modes. We show explicitly that extra degrees of freedom in a coupled cavity allow explorations of both parametric instability and cooling regimes with high parametric gain in a single table top experiment. This work can motivate experimental realizations of the three-mode parametric instability which might be an issue in next-generation gravitational-wave detectors with high optical-power cavities, helping in the development of better models, and in developing techniques for controls. In addition, we show that the same scheme can be implemented in the resolved-sideband acoustic-mode cooling.
\end{abstract}

DOI: 10.1103/PhysRevA.78.063809

PACS number(s): 42.65.Yj, 42.60.-v, 04.80.Nn

\section{INTRODUCTION}

Parametric interactions have wide applications and arouse great interests in various fields of physics, from highsensitivity transducers to low-noise amplifiers and optical parametric oscillators. Two-mode parametric interactions have been used to cool acoustic modes of mechanical oscillators. In Ref. [1], the authors cooled the normal mode of a 1.5 tonne $\mathrm{Nb}$ bar down to $5 \mathrm{mK}$ by using a microwave parametric transducer. With the same principle, a high-frequency acoustic mode of a nanomechanical oscillator is cooled near its quantum ground state through coupling to a superconducting single-electron transistor [2]. More recently, by coupling mechanical resonators to optical cavities, various table top experiments have demonstrated significant cooling of acoustic modes [3-13]. These experiments show great potential of achieving the quantum ground state of macroscopic mechanical oscillators, which would be a breakthrough in both theoretical and experimental physics.

Three-mode optoacoustic parametric interactions were first investigated by Braginsky et al. $[14,15]$ in the context of high optical-power Fabry-Perot cavities for interferometric gravitational-wave detectors. It was shown that three-mode interactions led to a risk of parametric instability (PI) in which the amplitude of acoustic modes of test masses could grow exponentially, thus undermining sensitivities of the detectors. The cause of parametric instability is the radiation pressure mediated coupling between optical modes and acoustic modes. It can occur if the shape of high-order cavity modes, hereafter denoted by $\mathrm{TEM}_{m n}$, have a substantial overlap with that of acoustic modes and simultaneously if the mode gap between $\mathrm{TEM}_{m n}$ and $\mathrm{TEM}_{00}$ is equal to acousticmode frequency up to an error of the linewidth of cavity modes. Further theoretical studies have followed up this pioneering work. Zhao et al. [16] extended their analysis and took into account three-dimensional (3D) structures of the optical and acoustic modes. Ju et al. [17] further considered the overall contributions from multiple optical modes, showing that multiple interactions increase the risk of instability. Gurkovsky et al. [18] analyzed PI in a signal-recycled (SR) interferometer and shows that the chance of PI was reduced due to the narrow linewidth of the SR interferometer. Addi- tionally, many ideas have been proposed to prevent PI, e.g., changing the radius of curvature (ROC) of the mirror [16], using an optical spring tranquilizer [19] or a ring damper [20]. In light of the above predictions, it is important to develop experimental techniques for their investigations. In recent progress, the University of Western Australia group demonstrated three-mode interactions in an $80 \mathrm{~m}$-long Fabry-Perot cavity [21]. They used a compensation plate as a thermal lens to tune the effective ROC of the mirror, which changes the Gouy phase or equivalently the mode gap, such that the resonant condition mentioned is satisfied. By capacitively exciting an acoustic mode to interact with the $\mathrm{TEM}_{00}$ mode, they observed resonance of a high-order optical mode at the predicted frequency. This experiment confirmed the principle of three-mode interactions, but due to low optical power and small overlap, it had not yet achieved selfsustained parametric instability or cooling. In this paper, we propose an alternative method of tuning the Gouy phase. It was first introduced by Mueller [22] as a means of designing a stable recycling cavity for next-generation gravitationalwave detectors. By adding a mirror and a lens onto a single cavity, which forms a coupled cavity, one can obtain any desired Gouy phase shift simply by adjusting relative positions of these optical components. In the following sections, we will show explicitly that a table top experiment can be set up, allowing observations of three-mode interactions with high parametric gain. This can help us better understand possible parametric instability in interferometric gravitationalwave detectors, and can also be applied, in general, as a design concept for optoacoustic parametric amplifiers, specifically to the resolved-sideband cooling $[23,24]$ of acoustic modes.

This paper is organized as follows: In Sec. II, we will review the theory of three-mode optoacoustic interactions. In Sec. III, we will analyze three-mode interactions in a coupled cavity. Finally, we will summarize our results in Sec. IV.

\section{REVIEW OF THREE-MODE OPTOACOUSTIC PARAMETRIC INTERACTION THEORY}

For the coherence of this article, we will briefly review the theory of three-mode optoacoustic parametric interac- 


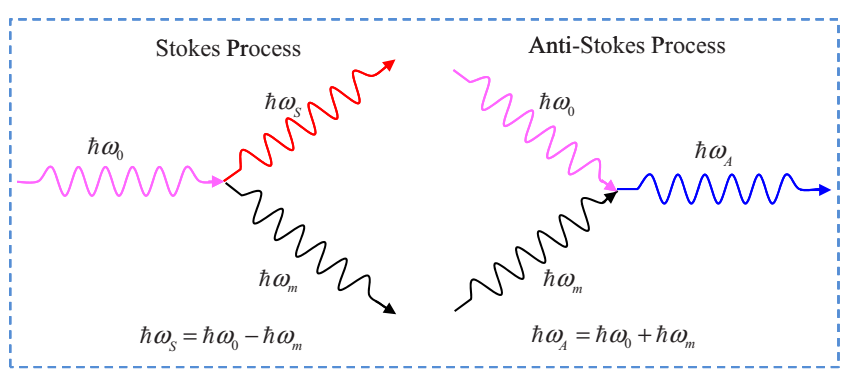

FIG. 1. (Color online) Parametric interactions between photons and phonons. In the Stokes process, the photon is down converted into a photon with lower energy and at the same time one phonon is created. This process will transfer energy from the optical field into the mechanical degree of freedom, thus amplifying the acoustic mode. In the anti-Stokes process, the photon is scattered into a higher-frequency photon, accompanied by annihilation of one phonon. In this process, the mechanical oscillation is damped. Classically, these two processes simply correspond to modulations of light by mechanical oscillations into two sidebands at frequencies $\omega_{0} \pm \omega_{m}$.

tions. They can be understood qualitatively in both quantum and classical pictures. In the former picture, we can treat the Fabry-Perot cavity as a multilevel quantum system occupied by photons, while the mechanical oscillator (end mirror) consists of phonon (acoustic) modes in different energy eigenstates. For simplicity, we only consider one phonon mode, which represents the situation in this proposed scheme. Initially, the photons stay in one eigenstate (the $\mathrm{TEM}_{00}$ mode). Resonant amplification occurs when the photons are scattered by the phonons into another eigenstate (the $\mathrm{TEM}_{m n}$ mode). The phonons can absorb energy from the photons (conventionally known as a Stokes process in Raman scattering), causing instability. Alternatively, they can release energy into the optical field (anti-Stokes process), which is also called cooling in the literature. We show both processes schematically in Fig. 1. In the classical picture [14], mechanical oscillations modulate the carrier frequency $\omega_{0}$ into two sidebands. The lower sideband corresponds to the Stokes mode with frequency $\omega_{S}=\omega_{0}-\omega_{m}$ and the upper sideband is the anti-Stokes mode with $\omega_{A}=\omega_{0}+\omega_{m}$. If they match the frequency of $\mathrm{TEM}_{m n} \omega_{1}$, the higher-order optical mode will be excited. In turn, these resonant optical modes TEM $_{m n}$ and $\mathrm{TEM}_{00}$ will exert a radiation pressure on the mechanical oscillator at the beating frequency $\left|\omega_{1}-\omega_{0}\right|=\omega_{m}$. Depending on the phase, this force will either amplify or damp the motion of the oscillator. To achieve a good coupling, a substantial spatial overlap between the acoustic mode and the $\mathrm{TEM}_{m n}$ mode is required.

To quantify the interactions, we follow the formalism in Ref. [14]. In the paper, they introduced parametric gain $\mathcal{R}$ to quantify the strength of three-mode interactions, which is defined as

$$
\mathcal{R}=\frac{4 I_{c} Q_{m}}{m c L \omega_{m}^{2}}\left[\frac{Q_{S} \Lambda_{S}}{1+\left(\Delta \omega_{S} / \gamma_{S}\right)^{2}}-\frac{Q_{A} \Lambda_{A}}{1+\left(\Delta \omega_{A} / \gamma_{A}\right)^{2}}\right] .
$$

Here subscript $S$ denotes Stokes mode and $A$ anti-Stokes mode, $L$ is the total length of the cavity, $I_{c}$ is the intracavity power, $m$ is the mass, and $Q_{m}$ is the mechanical quality factor. The detunings $\Delta \omega_{S(A)} \equiv\left|\omega_{S(A)}-\omega_{1}\right|$ and $\gamma_{A(S)}$ is decay rates of optical modes. Overlapping factor $\Lambda_{A(S)}$ is given by

$$
\Lambda_{A(S)}=\frac{V\left(\int f_{0}\left(\vec{r}_{\perp}\right) f_{A(S)}\left(\vec{r}_{\perp}\right) u_{z} d \vec{r}_{\perp}\right)^{2}}{\int\left|f_{0}\right|^{2} d \vec{r}_{\perp} \int\left|f_{A(S)}\right|^{2} d \vec{r}_{\perp} \int|\vec{u}|^{2} d V},
$$

where $f_{0, A, S}$ is optical-mode shapes and $u_{z}$ is the component of $\vec{u}$ normal to the mirror surface. The integrals $\int d \vec{r}_{\perp}$ and $\int d V$ correspond to integration over the mirror surface and volume $V$, respectively. For simplicity, we assume that $\Lambda_{S(A)} \sim 1$, which can be achieved in real experiments. In the tuned case that $\Delta \omega_{S(A)}=0, \mathcal{R}$ can be simply written as

$$
\mathcal{R}= \pm \frac{4 I_{c} Q_{m} Q_{S(A)}}{m c L \omega_{m}^{2}},
$$

with + for the Stokes process and - for the anti-Stokes process. The resulting decay rate of the acoustic mode $\gamma_{m}^{\prime}$ can be written as

$$
\gamma_{m}^{\prime}=\frac{1}{2}\left[\left(\gamma_{S(A)}+\gamma_{m}\right)-\sqrt{\left(\gamma_{S(A)}-\gamma_{m}\right)^{2}+4 \mathcal{R} \gamma_{S(A)} \gamma_{m}}\right] .
$$

Since usually $\gamma_{A(S)} \gg \gamma_{m}$, then

$$
\gamma_{m}^{\prime} \approx(1-\mathcal{R}) \gamma_{m}
$$

When $\mathcal{R}=0$, we obtain the trivial case $\gamma_{m}^{\prime}=\gamma_{m}$. For the Stokes process with $\mathcal{R}>0$ (positive gain), the decay rate of the acoustic mode decreases and the mechanical oscillation will be amplified. PI happens if $\mathcal{R}>1$, where we have $\gamma_{m}^{\prime}$ $<0$. For the anti-Stokes process with $\mathcal{R}<0$ (negative gain), the acoustic mode will be cooled as the effective decay rate increases.

\section{THREE-MODE INTERACTIONS WITH A COUPLED CAVITY}

In this section, we will discuss how to explore three-mode interactions using a coupled cavity. To make our analysis close to realistic experiments, we consider a torsional acoustic mode with frequency $\sim 1 \mathrm{MHz}$ interacting with the optical $\mathrm{TEM}_{10}$ and $\mathrm{TEM}_{00}$ modes. The configuration is chosen because $\mathrm{MHz}$ can be easily achieved in a mm-scale structure and the torsional mode has a large spatial overlap with the $\mathrm{TEM}_{10}$ mode.

To begin with, let us consider a single Fabry-Perot cavity to see why a coupled cavity is necessary. The free spectral range of a single cavity with length $\sim 10 \mathrm{~cm}$ is approximately $1 \mathrm{GHz}$. Therefore, as shown in Fig. 2, one has to build either a near-planar or near-concentric cavity to obtain a desired mode gap around $1 \mathrm{MHz}$ between $\mathrm{TEM}_{10}$ and $\mathrm{TEM}_{00}$. For both cases, the cavity is marginally stable and susceptible to misalignment. It is also difficult to obtain access to both instability and cooling regimes in a single setup. Creating a coupled Fabry-Perot cavity solves these problems. As we will show later, the resulting scheme is stable. Addi- 


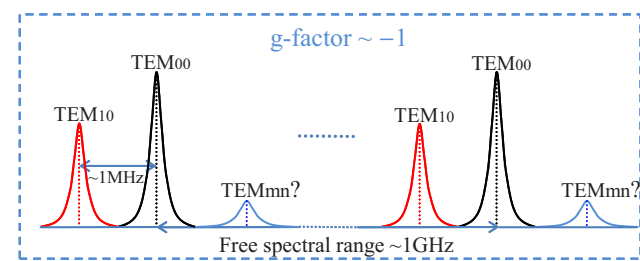

(a)

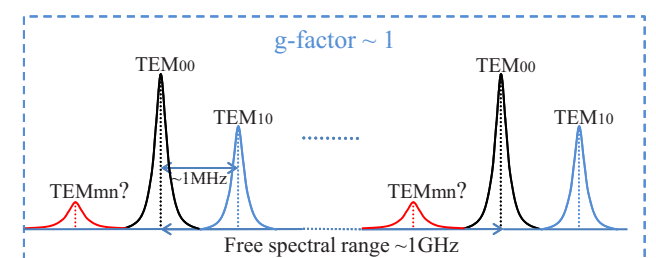

(b)

FIG. 2. (Color online) The optical modes of single Fabry-Perot cavity with length $\sim 10 \mathrm{~cm}$. (a) The mode distribution for a nearconcentric cavity with $g$ factor $\sim-1$ which is suitable for observing PI, while (b) is the near-planar case with $g$ factor $\sim 1$ which is suitable

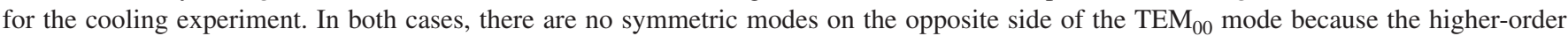
mode $^{\text {TEM }}{ }_{m n}$ marked with "?" are highly lossy due to diffraction losses. This is preferred for experimental realizations of three-mode interactions because we know from Eq. (1) that any symmetric mode on the opposite side of the $\mathrm{TEM}_{00}$ mode will reduce the absolute value of parametric gain. However, both cavities are marginally stable and very susceptible to misalignment.

tionally, we can easily tune between instability and cooling regimes. The coupled cavity is shown schematically in Fig. 3 . It is similar to the configuration of power or signal recycling interferometers $[25,26]$ when one considers either the common mode or the differential mode. The field dynamics can be easily obtained as shown in Ref. [27] by treating the subcavity as an effective mirror with frequency and modedependent transmissivity and reflectivity. Specifically, the effective transmissivity $t_{01}$ is

$$
t_{01} \equiv \frac{E_{12}}{E_{\text {in }}}=\frac{t_{0} t_{1}}{1+r_{0} r_{1} e^{i \delta \phi_{01}}}
$$

and the effective reflectivity $r_{10}$ is given by

$$
r_{10} \equiv \frac{E_{12}}{E_{21}}=-r_{1}-\frac{t_{1}^{2} r_{0} e^{i \delta \phi_{01}}}{1+r_{0} r_{1} e^{i \delta \phi_{01}}}
$$

The corresponding $E_{12}$ inside the main cavity can be written as

$$
E_{12}=\frac{E_{\mathrm{in}} t_{01}}{1+r_{10} r_{2} e^{i \delta \phi_{12}}}=\frac{E_{\mathrm{in}} t_{01}}{1-\left|r_{10}\right| r_{2} e^{i\left[\arg \left(r_{10}\right)+\delta \phi_{12}+\pi\right]}} .
$$

The resonance occurs when the phase factor in Eq. (8) is equal to $2 n \pi$, which critically depends upon the phase angle of the effective reflectivity, namely $\arg \left(r_{10}\right)$. Specifically, when the $\mathrm{TEM}_{00}$ mode resonates inside the main cavity, which requires that $\delta \phi_{01}^{\mathrm{TEM}_{00}}=\delta \phi_{12}^{\mathrm{TEM}_{00}}=2 n \pi$, the phase shift of the $\mathrm{TEM}_{10}$ mode $\delta \phi_{i j}^{\mathrm{TEM}_{10}}$ is

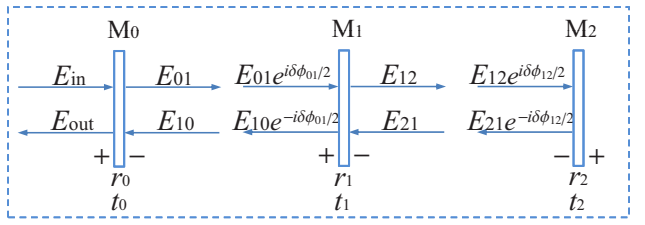

FIG. 3. (Color online) The optical fields of the coupled cavity. Here $\delta \phi_{01,12}$ are the round-trip phase shift of light in the subcavity (formed by $M_{0}$ and $M_{1}$ ) and main cavity (formed by $M_{1}$ and $M_{2}$ ), respectively. We use the convention that the mirrors have minus reflectivity on the side with a coating layer.

$$
\delta \phi_{i j}^{\mathrm{TEM}_{10}}=\frac{2 L_{i j}}{c} \Delta \omega-2 \Phi_{g}^{i j}+2 n^{\prime} \pi, \quad i j=01,12,
$$

where $\Delta \omega \equiv \omega_{1}-\omega_{0}$ is the mode gap between $\mathrm{TEM}_{10}$ and $\mathrm{TEM}_{00}, \Phi_{g}$ is the Gouy phase, and $n, n^{\prime}$ are integers. In order to satisfy the resonant condition for three-mode interactions, we need to adjust $\delta \phi_{01}^{\mathrm{TEM}_{10}}$, which changes $\arg \left(r_{10}\right)$, such that $\Delta \omega= \pm \omega_{m}$. To achieve this, one obvious way is to change the length of subcavity $L_{01}$, but this turns out to be impractical due to a small tuning range. An alternative and more practical approach, as shown in Fig. 4, is to add another lens or concave mirror inside the subcavity to tune the Gouy phase $\Phi_{g}^{01}$. The resulting scheme is similar to the proposed stable recycling cavity for next-generation gravitationalwave detectors [22]. With the additional lens, the Gaussian beam becomes focused inside the subcavity. Since the Gouy phase changes almost from $-\frac{\pi}{2}$ to $\frac{\pi}{2}$ within one Rayleigh range around the waist, one can easily obtain a desired $\delta \phi_{01}^{\mathrm{TEM}_{10}}$ simply by adjusting the position of $M_{0}$ near the waist. This might lead to problems with power density due to the small waist size, but for the table top experiment we consider here, the power density is quite low.

The corresponding $\Phi_{g}^{01}$ with an additional lens can be derived straightforwardly by using the ray transfer relation for a Gaussian beam, which is given by

$$
q^{\prime}=\frac{f q}{f-q} .
$$

Here $f$ is the focal length of $L_{1}, q^{\left({ }^{\prime}\right)} \equiv z^{\left({ }^{\prime}\right)}+i z_{R}^{\left({ }^{\prime}\right)}, z$ is the displacement relative to the waist, $z_{R}$ is the Rayleigh range,

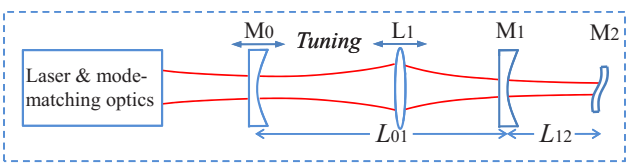

FIG. 4. (Color online) The optical layout for the table top experiment, where a $1 \mathrm{MHz}$ microtorsional oscillator $\left(M_{2}\right)$ interacts with the optical $\mathrm{TEM}_{10}$ mode and the $\mathrm{TEM}_{00}$ mode. By tuning the positions of mirror $M_{0}$ and lens $L_{1}$, we can continuously change the frequency of $\mathrm{TEM}_{10}$. If the losses in $L_{1}$ were an issue, it could easily be replaced by a concave mirror. 


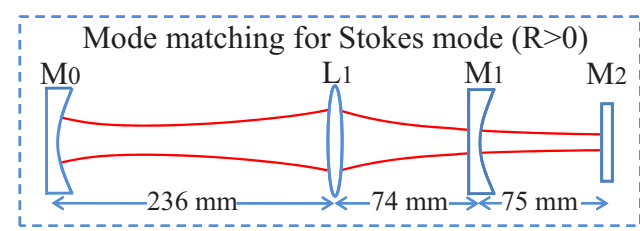

(a)

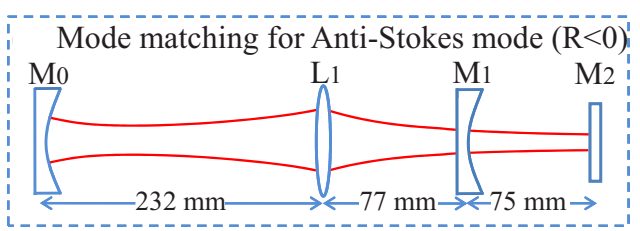

(b)

FIG. 5. (Color online) The mode matching for the positive gain and negative gain by adjusting the relative position of $M_{0}$ and $L_{1}$. Only small adjustment is needed to tune from one case to another.

and superscript ' denotes quantities after the lens. This dictates

$$
z^{\prime}=\frac{f\left(z f-z^{2}-z_{R}^{2}\right)}{(f-z)^{2}+z_{R}^{2}}
$$

$$
z_{R}^{\prime}=\frac{z_{R} f^{2}}{(f-z)^{2}+z_{R}^{2}}
$$

The resulting Gouy phase at any point is given by

$$
\Phi_{g}(z)= \begin{cases}\arctan \left(z / z_{R}\right), & z<z_{L}, \\ \arctan \left[\left(z-z_{L}+z_{L}^{\prime}\right) / z_{R}^{\prime}\right]+\arctan \left(z_{L} / z_{R}\right)-\arctan \left(z_{L}^{\prime} / z_{R}^{\prime}\right), & z \geqslant z_{L},\end{cases}
$$

where $z_{L}^{\left({ }^{\prime}\right)}$ is the position of $L_{1}$ relative to the waist. Gouy phase $\Phi_{g}^{01}$ is the difference between wave fronts at $M_{0}$ and $M_{1}$, namely

$$
\Phi_{g}^{01}=\Phi_{g}\left(z_{M_{0}}\right)-\Phi_{g}\left(z_{M_{1}}\right),
$$

where $z_{M_{0}}$ and $z_{M_{1}}$ are the positions of $M_{0}$ and $M_{1}$ relative to the waist, respectively. Therefore, by adjusting the positions of $M_{0}$ and $L_{1}$ as shown in Fig. 4, we can continuously tune $\Phi_{g}^{01}$ such that $\Delta \omega= \pm \omega_{m}$.

Equations (6)-(14) provide the design tools of the coupled cavity for three-mode interactions. To realize the experiment, we first need to design the main cavity and specify $L_{12}, \omega_{m}$, the ROCs of $M_{0}, M_{1}, M_{2}$, and the focal length of $L_{1}$. From Eq. (8) and Eq. (9), we can find out the required $\arg \left(r_{01}\right)$ which gives the right mode gap between $\mathrm{TEM}_{10}$ and $\mathrm{TEM}_{00}$. This will give us one constraint. Combining with the require-

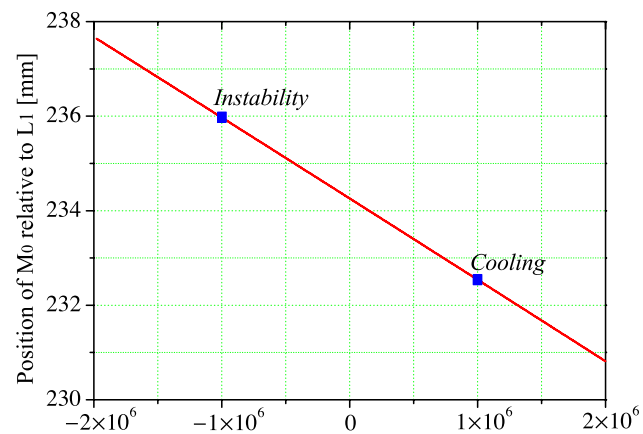

(a) Frequency difference between TEM 10 and TEM00 $[\mathrm{Hz}]$ ment of mode matching to $M_{0}$, we can fix two degrees of freedom of the system, namely, the positions of $M_{0}$ and $L_{1}$. To demonstrate this principle explicitly, we present a solution that is close to a realistic experimental setup. We assume the following:

$$
\begin{gathered}
L_{12}=75 \mathrm{~mm}, \quad \omega_{m}=1 \mathrm{MHz}, \quad f=100 \mathrm{~mm}, \\
R_{0}=500 \mathrm{~mm}, \quad r_{0}=\sqrt{0.999}, \quad A_{0}=500 \mathrm{ppm}, \\
R_{1}=100 \mathrm{~mm}, \quad r_{1}=\sqrt{0.9}, \quad A_{1}=500 \mathrm{ppm}, \\
R_{2}=\infty \mathrm{mm}, \quad r_{2}=\sqrt{0.9995}, \quad A_{2}=500 \mathrm{ppm} .
\end{gathered}
$$

Here $R_{i}(i=0,1,2)$ are ROCs, $r_{i}$ denotes the amplitude reflectivity, $t_{i}$ is the amplitude transmissivity, and $A_{i}$ is the optical loss which satisfy $r_{i}^{2}+t_{i}^{2}+A_{i}=1(i=0,1,2)$.

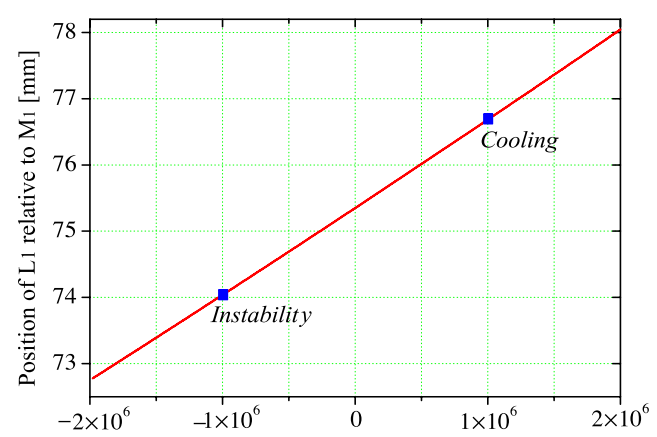

(b) Frequency difference between TEM 10 and TEM $00[\mathrm{~Hz}]$

FIG. 6. (Color online) Panels (a) and (b) show the mode gap between $\mathrm{TEM}_{10}$ and $\mathrm{TEM}_{00}$ as a function of position of $M_{0}$ relative to $L_{1}$ and position of $L_{1}$ relative to $M_{1}$, respectively. The dots in both figures are the situations considered in Fig. 5. Clearly, we can tune between the instability and cooling regimes continuously. 

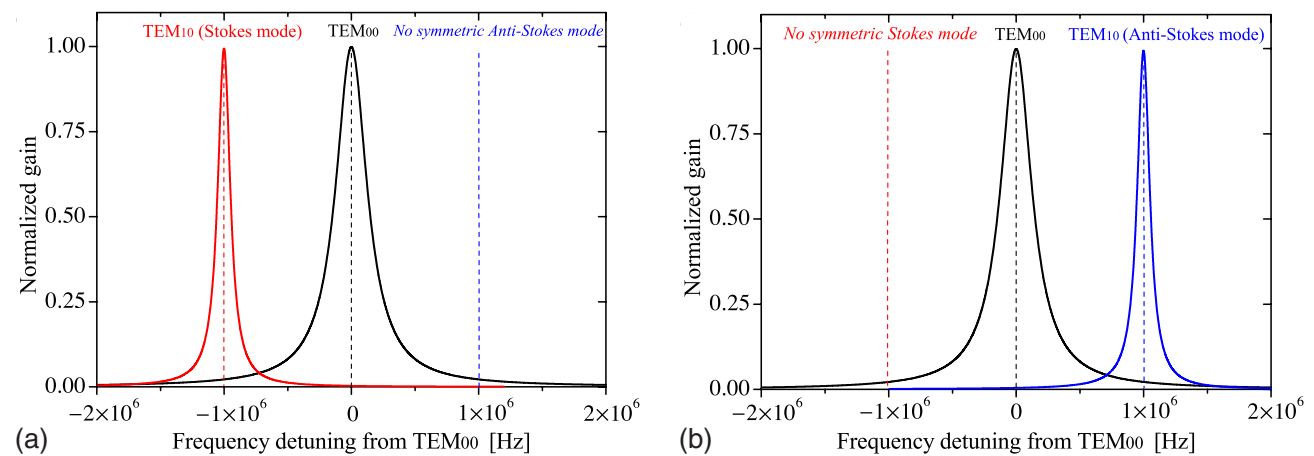

FIG. 7. (Color online) The normalized gain of the $\mathrm{TEM}_{00}$ mode and the $\mathrm{TEM}_{10}$ mode in the positive and negative gain configurations. The mode gap is equal to $\omega_{m} \sim 1 \mathrm{MHz}$, which fulfills the resonant condition for the three-mode optoacoustic interactions. Here we simply assume that the size of the mirrors is infinite so the quality factor of the TEM $\mathrm{T}_{10}$ mode is solely due to optical losses such as absorbtion. This assumption is reasonable when the mode number is small. Given the specifications in the main text, $Q_{A} \approx Q_{S}=2.4 \times 10^{9}$ and $\omega_{m} / \gamma_{A}<1$. Therefore, it can be implemented in the resolved-sideband cooling. (a) The $\mathrm{TEM}_{10}$ mode is $1 \mathrm{MHz}$ below the TEM $\mathrm{T}_{00}$ mode. (b) The TEM $_{10}$ mode is $1 \mathrm{MHz}$ above the $\mathrm{TEM}_{00}$ mode.

The results of mode matching for both positive (instability) and negative (cooling) gain configurations are shown in Fig. 5. In Fig. 6, we show the mode gap between $\mathrm{TEM}_{10}$ and $\mathrm{TEM}_{00}$ as a function of the position of $M_{0}$ relative to $L_{1}$ and the position of $L_{1}$ relative to $M_{1}$. In this particular case, the dependence is almost linear with a slope $\sim 2 \mathrm{~mm} / \mathrm{MHz}$ for both panels. This indicates that to tune within a cavity linewidth $\sim 0.1 \mathrm{MHz}$, it requires the mirror position to be adjusted within several $100 \mu \mathrm{m}$, which can be achieved easily. Therefore, we can continuously tune between instability and cooling regimes. Figure 7 shows the resulting resonance curves for both cases with the corresponding mode matching shown in Fig. 5. The corresponding mode gap between the $\mathrm{TEM}_{10}$ and $\mathrm{TEM}_{00}$ modes is equal to $\omega_{m} \sim 1 \mathrm{MHz}$. More importantly, there is no symmetric mode on the opposite side of the $\mathrm{TEM}_{00}$ mode, whose presence could contribute a parametric gain with the opposite sign, thereby suppressing the overall effects. The absolute value of parametric gain $\mathcal{R}$ could be larger than 1 , if we further assume that the intracavity power $I_{c}$ is $100 \mathrm{~mW}, Q_{m}=10^{6}$, the mass of the oscillator $m=1 \mathrm{mg}$, and the wavelength of light is $1064 \mathrm{~nm}$. Since the cavity is in the resolved-sideband regime where the cavity linewidth is much smaller than the mechanical frequency [23], this configuration can also be applied in the resolvedsideband cooling of acoustic modes, which is less susceptible to quantum noise. Although the quantum noise analysis in Refs. [23,24,28] only discusses a two-mode system, their results can be extended to the three-mode system by viewing the $\mathrm{TEM}_{00}$ mode as the carrier light. This can be justified by writing down the Hamiltonian of this system, which is

$$
\begin{aligned}
\hat{H}= & \frac{\hat{p}^{2}}{2 m}+\frac{1}{2} m \omega_{m}^{2} \hat{x}^{2}+\hbar \omega_{0} \hat{a}^{\dagger} \hat{a}+\hbar \omega_{1} \hat{b}^{\dagger} \hat{b} \\
& +\hbar G_{0} \hat{x}\left(\hat{a}^{\dagger} \hat{b}+\hat{b}^{\dagger} \hat{a}\right)+\hat{H}_{\text {drive }} .
\end{aligned}
$$

Here the first two terms represent the energy of the acoustic mode, where $\hat{x}$ is displacement and $\hat{p}$ is momentum which satisfy the commutation relation $[\hat{x}, \hat{p}]=i \hbar ; \hat{a}$ and $\hat{b}$ are the annihilation operators of the TEM 00 and $\mathrm{TEM}_{10}$ modes, respectively; the fifth term quantifies the interactions between the optical modes and the acoustic mode with the coupling constant $G_{0} \equiv \Lambda_{A, S} \omega_{0} / L$; the last term corresponds to external driving. Since only the $\mathrm{TEM}_{00}$ mode is pumped externally, the interaction term can be linearized as

$$
\hat{H}_{\mathrm{I}} \approx \hbar G_{0} \hat{x}\left(\bar{a}^{*} \hat{b}+\bar{a} \hat{b}^{\dagger}\right),
$$

which is the same interaction considered in Refs. [23,28] after linearizing. Therefore, this three-mode Hamiltonian can be mapped into an effective two-mode Hamiltonian by replacing $\hat{a}$ with its classical amplitude $\bar{a}$. All the conclusions reached in the two-mode case are also valid here. The only difference is that the carrier light $\mathrm{TEM}_{00}$ is also on resonance rather than far detuned in the two-mode case [23,28]. Therefore, we can achieve the resolved-sideband limit without compromising intracavity optical power. Also, the resonant condition $\omega_{1}-\omega_{0}=\omega_{m}$ here is equivalent to the frequency detuning equal to $\omega_{m}$ in the two-mode case. This optimizes the energy transfer from the acoustic mode to the optical fields in the resolved-sideband limit $[23,28]$.

\section{CONCLUSION}

We have shown that a table top experiment based on a coupled cavity can be set up to realize self-sustained threemode optoacoustic parametric interactions. Small adjustments of the positions of the optical components enable us to continuously tune between instability and cooling regimes. This relatively simple scheme can be applied to design optoacoustic amplifiers and experimentally investigate the three-mode parametric instability, a possible issue of nextgeneration gravitational-wave detectors with high opticalpower cavities. Besides, it can also be used to realize the resolved-sideband cooling of acoustic modes with threemode interactions.

\section{ACKNOWLEDGMENTS}

We thank all the participants attending the Parametric Instability Workshop held at the Australian International 
Gravitational Observatory (AIGO) site, especially Professor S. P. Vyatchanin and Dr. S. E. Strigin for stimulating discussions and giving precious advice on the improvement of the draft. We thank D. Price for pointing out several errors in the manuscripts. H.M. thanks Professor Y. Chen for the invitation to visit the Albert-Einstein-Institut. The visit was supported by the Alexander von Humboldt
Foundation's Sofja Kovalevskaja Programme. This research has been supported by the Australian Research Council and the Department of Education, Science and Training and by the U.S. National Science Foundation. We thank the LIGO Scientific Collaboration International Advisory Committee of the Gingin High Optical Power Facility for their support.
[1] D. G. Blair, E. N. Ivanov, M. E. Tobar, P. J. Turner, F. van Kann, and I. S. Heng, Phys. Rev. Lett. 74, 1908 (1995).

[2] A. Naik, O. Buu, M. D. LaHaye, A. D. Armour, A. A. Clerk, M. P. Blencowe, and K. C. Schwab, Nature (London) 443, 193 (2006).

[3] S. Gigan, H. R. Böhm, M. Paternostro, F. Blaser, G. Langer, J. B. Hertzberg, K. C. Schwab, D. Bäuerle, M. Aspelmeyer, and A. Zeilinger, Nature (London) 444, 67 (2006).

[4] O. Arcizet, P. F. Cohandon, T. Briant, M. Pinard, and A. Heidmann, Nature (London) 444, 71 (2006).

[5] D. Kleckner and D. Bouwmeester, Nature (London) 444, 75 (2006).

[6] A. Schliesser, P. DelHaye, N. Nooshi, K. J. Vahala, and T. J. Kippenberg, Phys. Rev. Lett. 97, 243905 (2006).

[7] T. Corbitt, Y. Chen, E. Innerhofer, H. Muller-Ebhardt, D. Ottaway, H. Rehbein, D. Sigg, S. Whitcomb, C. Wipf, and N. Mavalvala, Phys. Rev. Lett. 98, 150802 (2007).

[8] T. Corbitt, C. Wipf, T. Bodiya, D. Ottaway, D. Sigg, N. Smith, S. Whitcomb, and N. Mavalvala, Phys. Rev. Lett. 99, 160801 (2007).

[9] A. Schliesser, R. Rivière, G. Anetsberger, O. Arcizet, and T. J. Kippenberg, e-print arXiv:0709.4036v1.

[10] M. Poggio, C. L. Degen, H. J. Mamin, and D. Rugar, Phys. Rev. Lett. 99, 017201 (2007).

[11] J. D. Thompson, B. M. Zwickl, A. M. Jayich, F. Marquardt, S. M. Girvin, and J. G. E. Harris, Nature (London) 452, 72 (2008).

[12] C. M. Mow-Lowry, A. J. Mullavey, S. Goßler, M. B. Gray, and D. E. McClelland, Phys. Rev. Lett. 100, 010801 (2008).

[13] S. W. Schediwy, C. Zhao, L. Ju, D. G. Blair, and P. Willems,
Phys. Rev. A 77, 013813 (2008).

[14] V. B. Braginsky, S. E. Strigin, and S. P. Vyatchanin, Phys. Lett. A 287, 331 (2001).

[15] V. B. Braginsky, S. E. Strigin, and S. P. Vyatchanin, Phys. Lett. A 305, 111 (2002).

[16] C. Zhao, L. Ju, J. Degallaix, S. Gras, and D. G. Blair, Phys. Rev. Lett. 94, 121102 (2005).

[17] L. Ju, S. Gras, C. Zhao, J. Degallaix, and D. G. Blair, Phys. Lett. A 354, 360 (2006).

[18] A. G. Gurkovsky, S. E. Strigin, and S. P. Vyatchanin, Phys. Lett. A 362, 91 (2007).

[19] V. B. Braginsky and S. P. Vyatchanin, Phys. Lett. A 293, 228 (2002).

[20] S. Gras, D. G. Blair, and L. Ju, Phys. Lett. A 372, 1348 (2008).

[21] C. Zhao, L. Ju, Y. Fan, S. Gras, B. J. J. Slagmolen, H. Miao, P. Barriga, D. G. Blair, D. J. Hosken, A. F. Brooks, P. J. Veitch, D. Mudge, and J. Munch, Phys. Rev. A 78, 023807 (2008).

[22] G. Mueller, LIGO Report No. G070441-00-R (2007).

[23] F. Marquardt, J. P. Chen, A. A. Clerk, and S. M. Girvin, Phys. Rev. Lett. 99, 093902 (2007).

[24] I. Wilson-Rae, N. Nooshi, W. Zwerger, and T. J. Kippenberg, Phys. Rev. Lett. 99, 093901 (2007).

[25] B. J. Meers, Phys. Rev. D 38, 2317 (1988).

[26] A. Buonanno and Y. Chen, Phys. Rev. D 64, 042006 (2001).

[27] M. Rakhmanov, Ph.D. thesis, California Institute of Technology, 2000.

[28] C. Genes, D. Vitali, P. Tombesi, S. Gigan, and M. Aspelmeyer, Phys. Rev. A 77, 033804 (2008). 